\title{
Simultaneous Iterative Boltzmann Inversion for Coarse-Graining of Polyurea
}

Vipin Agrawal, ${ }^{\dagger}$ Gaurav Arya, ${ }^{\dagger}$ and Jay Oswald ${ }^{\dagger * *}$

${ }^{\dagger}$ School for the Engineering of Matter, Transport and Energy, Arizona State University, Tempe, Arizona 85287, United States

${ }^{\ddagger}$ Department of NanoEngineering, University of California San Diego, La Jolla, California 92093, United States

ABSTRACT: Polyurea is an alternating copolymer with excellent viscoelastic properties for dissipating shock and impact loads; however, a molecular-level understanding of how its chemistry relates to its performance remains elusive. While molecular dynamics simulations can in theory draw connections between molecular structure and viscoelastic properties, in practice the long relaxation times associated with polymer
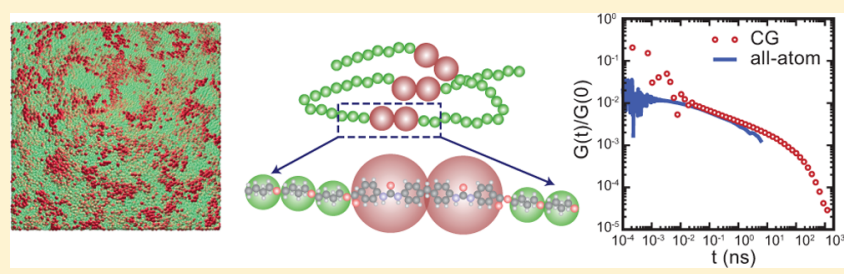
dynamics make such calculations prohibitively expensive. To address this issue, we have developed a coarse-grained (CG) model of polyurea in which each of the phenylmethaneaminobenzoate and tetramethylene-oxide units making up the polyurea chains are treated using individual CG beads. The parameters for the intra- and intermolecular force field of the CG model have been obtained in a rigorous manner by using the iterative Boltzmann inversion approach. We have validated the CG model against densities, heat capacities, and chain conformations obtained from fully atomistic MD simulations for oligomeric polyurea chains. A time-dependent dynamic rescaling method is proposed that allows for quantitative predictions of stress relaxation beyond microsecond time scales. The CG model introduced here opens up avenues to study the molecular structure-function relationship of polyurea and polyureabased materials.

\section{INTRODUCTION}

Polyurea is an elastomeric, alternating copolymer synthesized from the reaction between a difunctional isocyanate and a difunctional amine. Typically, the isocyanate is based on an aromatic moiety and the amine is based on a polyether, resulting in a polyurea chain with alternating regions of long, flexible, aliphatic segments and short, rigid, aromatic segments. ${ }^{1,2}$ Because of differences in the chemical interactions of the two types of segments, polyurea undergoes microphase segregation to yield hard domains, comprised primarily of ordered aromatic segments, surrounded by a soft, viscoelastic matrix, composed of mostly aliphatic segments. Such structured morphology seems to endow polyurea with excellent dissipative properties, making polyurea a useful material for shock-resistant coatings and reinforced composites. ${ }^{2}$ More recently, polyurea has found potential applications in protective gear of soldiers for mitigating shocks from blasts and ballistic impacts to prevent traumatic brain injury. ${ }^{3-5}$ This has spawned a renewed interest in polyurea, especially in resolving the molecular origin of its superior dissipative properties and using this knowledge to design new, improved shock mitigating materials.

While experiments have provided many invaluable insights into the dynamical response of polyurea, relating the observed response to the chemical composition, molecular architecture, and microstructure of polyurea remains a formidable task for experimentalists. Atomistic molecular dynamics (MD) simulations provide a powerful route to developing such a molecularlevel understanding. However, to capture the viscoelastic behavior of polyurea, the simulation domain must be larger than the microphase-separated domains in polyurea, which have thicknesses on the order of $10 \mathrm{~nm}$ in width and are longer in length. ${ }^{6}$ Even for intermediate-length homopolymers, the radius of gyration introduces a length scale longer than tens of nanometers. ${ }^{7}$ At the same time, the time scale of the simulation must exceed the longest relaxation mode in the system, typically the rotational or diffusional relaxation time in case of polymers. This characteristic time already exceeds tens of microseconds for short-chain polyethylene melts ${ }^{8}$ and is expected to be much longer in the case of polyurea. Since atomistic MD simulations are generally limited to systems sizes smaller than a few nanometers and time scales on the order of a few nanoseconds, computing the entire viscoelastic response of polyurea spanning low to high frequencies becomes nearly impossible, though the simulations can be used to probe localized, shorttime scale dynamics.

To overcome this challenge in simulating polyurea at realistic time and length scales, several groups ${ }^{9-11}$ have employed coarse-grained (CG) models of polyurea chains in which monomers or groups of contiguous monomers are represented as single CG beads, allowing chain conformations to be described by far fewer degrees of freedom. These models have allowed researchers to simulate the dynamics of sufficiently large polyurea systems for relevant time scales, yielding insights into the morphology and dynamics of polyurea, including the

Received: February 11, 2014

Revised: April 9, 2014

Published: May 7, 2014 
effects of hard-to-soft segment ratio and segregation strength on polyurea microstructure; ${ }^{11}$ the role of interconnected, deformable hard domains, arising from its alternating chain architecture, in shock mitigation; and the differences in the mechanical properties of hard and soft domains. ${ }^{10}$ However, the CG models of polyurea used so far are similar to the bead spring models pioneered by Kremer and Grest ${ }^{12}$ and therefore are not quantitatively predictive, as they have not been parametrized in a rigorous manner, e.g., to match the structural and dynamical features of polyurea computed from atomistic MD simulations, and the models have also not been validated against experimental measurements. Thus, these CG models can provide only qualitative and not quantitative predictions of the dynamical behavior of polyurea. For a comparison of systematically coarse-grained methods with other multiscale modeling methods for polymers, the reader is referred to the recent review article by $\mathrm{Li}$ et al. ${ }^{13}$

In this paper, we develop a systematic approach for coarsegraining polyurea by using the iterative Boltzmann inversion (IBI) approach. ${ }^{14-16}$ The approach involves simultaneous, iterative optimization of the interaction potentials between CG beads of the model until specific structural distributions derived from the CG model match the corresponding distributions computed from atomistic MD simulations. The resulting CG model reproduces quantitatively the density, structural configuration, and heat capacity of a melt of oligomeric polyurea obtained from atomistic MD simulations, but at orders of magnitude lesser computational effort. In addition, we introduce a time-dependent dynamic rescaling factor based on the ratio of the mean squared displacements of atomistic and CG oligomer systems that accounts for the faster dynamics of CG models of polymers. With this scaling factor, CG simulations quantitatively match their $\mathrm{MD}$ counterparts in predicting the stress relaxation spectrum of oligomeric polyurea. The CG model of polyurea and dynamic rescaling method developed and tested here will be used in the future to provide rapid predictions of the viscoelastic properties of polyurea-based composites as a function of chain architecture and composition, facilitating materials discovery and development efforts in the field of polyurea-based shock-dissipating materials.

\section{METHODS}

To develop an accurate coarse-grained (CG) model of polyurea, we implement the iterative Boltzmann inversion (IBI) method, ${ }^{14-16}$ previously used successfully to coarse-grain a variety of other polymers. ${ }^{4,17-20}$ Briefly, this approach treats the atomistic polymer chain as a chain of CG beads, where each bead represents a group of atoms along the polymer chain, allowing polymer chains to be described by significantly fewer degrees of freedom. The CG beads interact with each other, both along and across chains, through "effective" potentials derived in a rigorous, iterative manner such that the CG bead-chain exhibits similar configurational properties as the fully atomistic polymer chain; in other words, the CG bead-chain reproduces the structural distributions of the atomistic chains obtained from molecular dynamics (MD) simulations.

Coarse-Grained Model of Polyurea. Our model system is an idealization of a polyurea relevant to shock mitigation that is synthesized from a reaction between a diphenylmethane diisocyanate and a poly(tetramethylene oxide) diaminobenzoate. The resulting chain is an alternating copolymer made up of regions of hard segments composed of the four aromatic moieties plus the urea linkage and soft segments composed of the poly(tetramethylene oxide) chain (Figure 1a). Because of differences in the physical properties of the hard and soft segments, we use two distinct CG beads in representing such a chain. One type of bead, which we term soft bead, or " $S$ " for short, represents

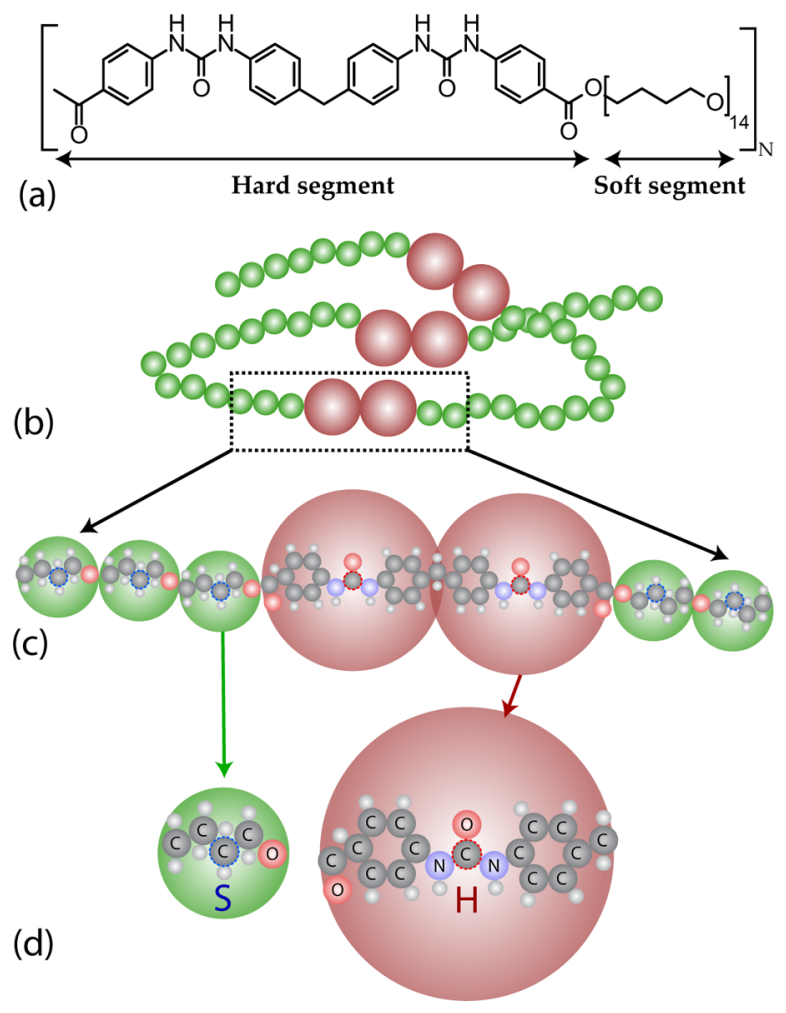

Figure 1. Coarse grained (CG) model of polyurea: (a) chemical structure of the repeating units of polyurea consisting of alternate hard and soft segments, (b) figurative representation of a short polyurea chain composed of coarse-grained beads, (c) coarse-grained mapping of hard and soft segments of polyurea, and (d) chemical structure of CG mapped beads; $S$ represents the soft bead while $H$ represents the hard bead. The carbon atoms within each bead that are encircled by dotted lines are the virtual sites from which interbead distances are calculated in the generation of structural distribution functions.

each of the 14 repeating $-\mathrm{C}_{4} \mathrm{H}_{8} \mathrm{O}$ - units in the soft segment (Figure 1d). A second type of bead, which we term hard bead or " $H$ ", represents each of the 2 symmetric halves $-\mathrm{C}_{6} \mathrm{H}_{6}-\mathrm{NH}-\mathrm{CO}-\mathrm{NH}-\mathrm{C}_{6} \mathrm{H}_{6}-\mathrm{CO}-$ of the hard segment (Figure 1d). The resulting CG bead-chain can therefore be denoted by $\left(\mathrm{H}_{2} \mathrm{~S}_{14}\right)_{n}$, where $n$ denotes the number of times the $\mathrm{H}_{2} \mathrm{~S}_{14}$ block is repeated, depending on the degree of polymerization of the polyurea chains.

Many of the recent experimental polyurea studies ${ }^{6,21-24}$ were conducted with material samples based on a modified MDI polycarbodiimide-modified diphenylmethane diisocyanate (Isonate $143 \mathrm{~L} / 2143 \mathrm{~L}$ ) and an oligomeric diamine (Versalink P1000). The multifunctional isocyanate introduces chemical cross-linking in polyurea through a small fraction of trifunctional polycarbodiimide adducts present in the modified MDI mixture. The percentage of trifunctional isocyanate is not well characterized, with reported values of isocyanate functionality varying from 2.1 to $2.4{ }^{21,25}$ In this work, trifunctional groups are not included so that all polyurea chains are purely linear without any physical cross-links. We will address the influence of trifunctional hard domain content in a future study.

Each CG bead type is assigned a mass equal to the sum of the molecular weights of its atomic constituents $\left(m_{H}=245 \mathrm{~g} / \mathrm{mol}\right.$ and $m_{S}=72 \mathrm{~g} / \mathrm{mol}$ ). The total potential energy of the CG bead-chain $U_{\text {tot }}$ is given by three contributions:

$$
U_{\text {tot }}=U_{\text {str }}+U_{\text {bend }}+U_{\text {nonb }}
$$

where $U_{\text {str }}$ is the energy associated with stretching the bonds between adjacent beads along the bead-chain, $U_{\text {bend }}$ is the energy associated with bending the angles subtended by consecutive bonds, and $U_{\text {nonb }}$ is the energy of nonbonded interactions between beads, on separate chains or beads located further than two bonds apart on the same 
chain. Many studies include a fourth contribution arising from the dihedral angles defined by four consecutive beads along the chain. However, we find that such a contribution is not required, as explained later.

The total bond stretching energy $U_{\text {str }}$ is calculated as the sum of stretching energies of the three types of bonds present in the CG system:

$$
U_{\mathrm{str}}=\sum_{i=1}^{N_{\mathrm{H}-\mathrm{H}}} \mathcal{V}_{\mathrm{H}-\mathrm{H}}^{\mathrm{str}}\left(l_{i}\right)+\sum_{i=1}^{N_{\mathrm{H}-\mathrm{S}}} \mathcal{V}_{\mathrm{H}-\mathrm{S}}^{\mathrm{str}}\left(l_{i}\right)+\sum_{i=1}^{N_{\mathrm{S}-\mathrm{S}}} \mathcal{V}_{\mathrm{S}-\mathrm{S}}^{\mathrm{str}}\left(l_{i}\right)
$$

where $l_{i}$ is the length of the $i$ th bond; $\mathcal{V}_{\mathrm{H}-\mathrm{H}}^{\mathrm{str}}, \mathcal{V}_{\mathrm{H}-\mathrm{S}}^{\mathrm{str}}$, and $\mathcal{V}_{\mathrm{S}-\mathrm{S}}^{\mathrm{str}}$ are the CG potentials describing the stretching energies of the $H-H, H-S$, and $S-S$ bonds as a function of their lengths, respectively; and $N_{\mathrm{H}-\mathrm{H}}$, $N_{\mathrm{H}-\mathrm{S}}$, and $N_{\mathrm{S}-\mathrm{S}}$ are the number of bonds of each type present in the system.

Similarly, the net bending energy $U_{\text {bend }}$ has contributions arising from the three types of bond angles present in the CG model:

$$
\begin{aligned}
U_{\text {bend }} & =\sum_{i=1}^{N_{\mathrm{H}-\mathrm{H}-\mathrm{S}}} \mathcal{V}_{\mathrm{H}-\mathrm{H}-\mathrm{S}}^{\mathrm{bend}}\left(\theta_{i}\right)+\sum_{i=1}^{N_{\mathrm{H}-\mathrm{S}-\mathrm{S}}} \mathcal{V}_{\mathrm{H}-\mathrm{S}-\mathrm{S}}^{\text {bend }}\left(\theta_{i}\right) \\
+ & \sum_{i=1}^{N_{\mathrm{S}-\mathrm{S}-\mathrm{S}}} \mathcal{V}_{\mathrm{S}-\mathrm{S}-\mathrm{S}}^{\text {bend }}\left(\theta_{i}\right)
\end{aligned}
$$

where $\theta_{i}$ is the angle between the $i^{\text {th }}$ set of consecutive bonds; $\mathcal{V}_{\mathrm{H}-\mathrm{H}-\mathrm{S}}^{\text {bend }}, \mathcal{V}_{\mathrm{H}-\mathrm{S}-\mathrm{S}}^{\text {bend }}$, and $\mathcal{V}_{\mathrm{S}-\mathrm{S}-\mathrm{S}}^{\text {bend }}$ are the CG potentials describing the energy of bending the angles subtended by $H-H-S, H-S-S$, and $S-S-S$ segments as a function of their bending angles; and $N_{\mathrm{H}-\mathrm{H}-\mathrm{S}}$, $N_{\mathrm{H}-\mathrm{S}-\mathrm{S}}$, and $N_{\mathrm{S}-\mathrm{S}-\mathrm{S}}$ are the number of bending angles of each type present in the system.

Finally, the total nonbonded energy $U_{\text {nonb }}$ is calculated as the sum of all nonbonded interactions in between and across the $H$ and $S$ beads in the CG system:

$$
\begin{aligned}
U_{\text {nonb }}= & \sum_{i=1}^{N_{\mathrm{H}^{-1}}} \sum_{j=i+1}^{N_{\mathrm{H}}} \mathcal{V}_{\mathrm{H} / \mathrm{H}}^{\mathrm{nonb}}\left(r_{i j}\right)+\sum_{i=1}^{N_{\mathrm{H}}} \sum_{j=1}^{N_{\mathrm{S}}} \mathcal{V}_{\mathrm{H} / \mathrm{S}}^{\mathrm{nonb}}\left(r_{i j}\right) \\
& +\sum_{i=1}^{N_{\mathrm{S}^{-1}}} \sum_{j=i+1}^{N_{\mathrm{S}}} \mathcal{V}_{\mathrm{S} / \mathrm{S}}^{\mathrm{nonb}}\left(r_{i j}\right)
\end{aligned}
$$

where $r_{i j}$ is the distance between beads $i$ and $j ; \mathcal{V}_{\mathrm{H} / \mathrm{H}}^{\mathrm{nonb}}, \mathcal{V}_{\mathrm{H} / \mathrm{S}}^{\mathrm{nonb}}$, and $\mathcal{V}_{\mathrm{S} / \mathrm{S}}^{\mathrm{nonb}}$ are the CG potentials describing the nonbonded interactions between $H$ beads, between $H$ and $S$ beads, and between $S$ beads as a function of their separation distance, respectively; and $N_{\mathrm{H}}$ and $N_{\mathrm{S}}$ are the total number of $H$ and $S$ beads in the CG system, respectively.

The CG force field described by eqs 1-4 requires a total of 9 effective CG potentials to fully describe the energetics and conformational dynamics of the CG polyurea system. Next, we describe a procedure for deriving functional (or numerical) forms of these CG potentials, via a combination of atomistic and CG simulations.

Atomistic MD Simulations. The purpose of these simulations is to generate reference structural distributions of polyurea chains in the melt state for parametrizing the effective potentials of the CG model described above. Because simulations of full-length polymer systems are computationally prohibitive, CG potentials are usually derived from simulations of much shorter, oligomeric versions of the chains carried out at the same temperature and pressure. The assumption is that the potentials derived for shorter chains are transferable to fulllength chains. In this study, we use short atomistic chains of polyurea containing a single repeating motif (atomistic $\mathrm{H}_{2} \mathrm{~S}_{14}$ ) for generating the structural distributions.

We measure three types of structural distributions from our atomistic simulations. First, we collect distributions in bond lengths $l$, i.e., distances between virtual points along the atomistic polymer chain representing the centers of adjacent beads in the CG model. There are three types of bonds in our CG system, so we obtain three sets of bond length distributions, denoted by $P_{\mathrm{H}-\mathrm{H}}(l), P_{\mathrm{H}-\mathrm{S}}(l)$, and $P_{\mathrm{S}-\mathrm{S}}(l)$ for the $H-H, H-S$, and $S-S$ bonds. These distributions are used to parametrize the bond stretching potentials $\mathcal{V}_{\mathrm{H}-\mathrm{H}}^{\mathrm{str}}(l), \mathcal{V}_{\mathrm{H}-\mathrm{S}}^{\mathrm{str}}(l)$, and $\mathcal{V}_{\mathrm{S}-\mathrm{S}}^{\text {str }}(l)$ of our CG model. Second, we collect distributions in bond angles $\theta$, i.e., the angles between points along the atomistic chain representing the centers of three adjacent beads in the CG model. We thus obtain three distributions, $P_{\mathrm{H}-\mathrm{H}-\mathrm{S}}(\theta), P_{\mathrm{H}-\mathrm{S}-\mathrm{S}}(\theta), P_{\mathrm{S}-\mathrm{S}-\mathrm{S}}(\theta)$, corresponding to the three types of bond angles present in our CG chains, as defined by $H-H-S, H-S-S$, and $S-S-S$ bead triplets. These three distributions will be used to parametrize the three CG bending potentials $\mathcal{V}_{\mathrm{H}-\mathrm{H}-\mathrm{S}}^{\text {bend }}(\theta), \mathcal{V}_{\mathrm{H}-\mathrm{S}-\mathrm{S}}^{\text {bend }}(\theta)$, and $\mathcal{V}_{\mathrm{S}-\mathrm{S}-\mathrm{S}}^{\text {bend }}(\theta)$. Finally, we gather radial distribution functions (RDFs) of distances $r$ between pairs of points on the atomistic chains representing nonbonded centers of beads in the CG model. Three types of RDFs, namely, $g_{\mathrm{H} / \mathrm{H}}(r)$, $g_{\mathrm{H} / S}(r)$, and $g_{S / S}(r)$, are collected corresponding to distributions in distances between nonbonded pairs of $H$ beads, nonbonded $H$ and $S$ beads, and nonbonded pairs of $S$ beads, respectively. These $\mathrm{RDF}$ are used to parametrize the CG nonbonded potentials $\mathcal{V}_{\mathrm{H} / \mathrm{H}}^{\mathrm{nonb}}(r)$, $\mathcal{V}_{\mathrm{H} / \mathrm{S}}^{\mathrm{nonb}}(r)$, and $\mathcal{V}_{\mathrm{S} / \mathrm{S}}^{\mathrm{nonb}}(r)$. We also measure distributions in the dihedral angle, as defined by four points along the atomistic chain representing the centers of four adjacent beads in the CG bead-chain. However, these distributions measured for the different kinds of bead quadruplets (e.g., $H-H-S-S, H-S-S-S$, etc.) are quite flat, suggesting that a dihedral potentials might not be required in the CG force field of polyurea chains.

To perform MD simulations of the atomistic polyurea segments, we construct cubic simulation domains with edge lengths of $36.3 \AA$, each containing 20 randomly distributed chains of atomistic $\mathrm{H}_{2} \mathrm{~S}_{14}$ polyurea segments. For this purpose, we use the amorphous cell component within the Materials Studio environment. ${ }^{26}$ Simulations are carried out using the large-scale atomic/molecular massively parallel simulator (LAMMPS) package, developed at Sandia National Laboratories ${ }^{27}$ to integrate atomic trajectories with a time step of $1 \mathrm{fs}$. The atomic interactions are governed by the condensed-phase optimized molecular potentials for atomistic simulations studies (COMPASS) force field, a class II force field that is parametrized for a wide range of experimental measurements for organic compounds; ${ }^{28}$ the functional form of class II potentials can be found in various sources. ${ }^{27,29,30}$ Additionally, we use the particle-particle/particle-mesh (PPPM) approach in the LAMMPS $\mathrm{k}$-space package ${ }^{27}$ to calculate long-range Coulombic interactions.

To speed up equilibration of the initial randomly generated configurations, each atomistic system is equilibrated in a constant pressuretemperature (NPT) ensemble for $8 \mathrm{~ns}$ at an elevated temperature $(T=$ $500 \mathrm{~K})$ and atmospheric pressure and subsequently quenched to $300 \mathrm{~K}$ over a time span of $8 \mathrm{~ns}$. The systems are then held for an additional $16 \mathrm{~ns}$ at ambient temperature and pressure $(T=300 \mathrm{~K}$ and $p=1 \mathrm{~atm})$ so that the total simulation time for equilibration is $32 \mathrm{~ns}$. Following equilibration, a production run of $1 \mathrm{~ns}$ is performed in the canonical $(N V T)$ ensemble at $T=300 \mathrm{~K}$ for sampling pair, bond, and bond-angle structural distributions. To reduce the influence of the initial randomly generated state of each system the pair, bond, and bond-angle structural distributions are averaged from MD trajectories obtained from 15 different starting configurations. The average density over all runs is $1.068 \mathrm{~g} / \mathrm{cm}^{3}$, close to the experimentally measured density of polyurea. For calculating structural distributions, we use the carbon atom near the center of the backbone atoms of the relevant segments $(H$ or $S)$ as the CG bead center.

For the IBI method to generate accurate CG potentials, proper equilibration of the atomistic systems is critically important. Parts a-c of Figures 2 show the evolution of the radial distribution functions $g_{\mathrm{H} / \mathrm{H}}(r), g_{\mathrm{H} / \mathrm{S}}(r)$, and $g_{\mathrm{S} / \mathrm{S}}(r)$ for the three bead pairs at various equilibration times. In particular, $g_{\mathrm{H} / \mathrm{H}}(r)$ and $g_{\mathrm{H} / \mathrm{s}}(r)$ undergo significant changes before approaching a stationary state at $32 \mathrm{~ns}$ of equilibration. The convergence of $g_{\mathrm{H} / \mathrm{H}}(r)$ is more clearly shown by examining the time evolution of its peak value (Figure $2 \mathrm{~d}$ ). As smaller peak values in the radial distribution functions will lead to weaker attractive energies in the effective CG models, sampling an insufficiently equilibrated all-atom system will lead to under-represented attractive forces between CG beads. 

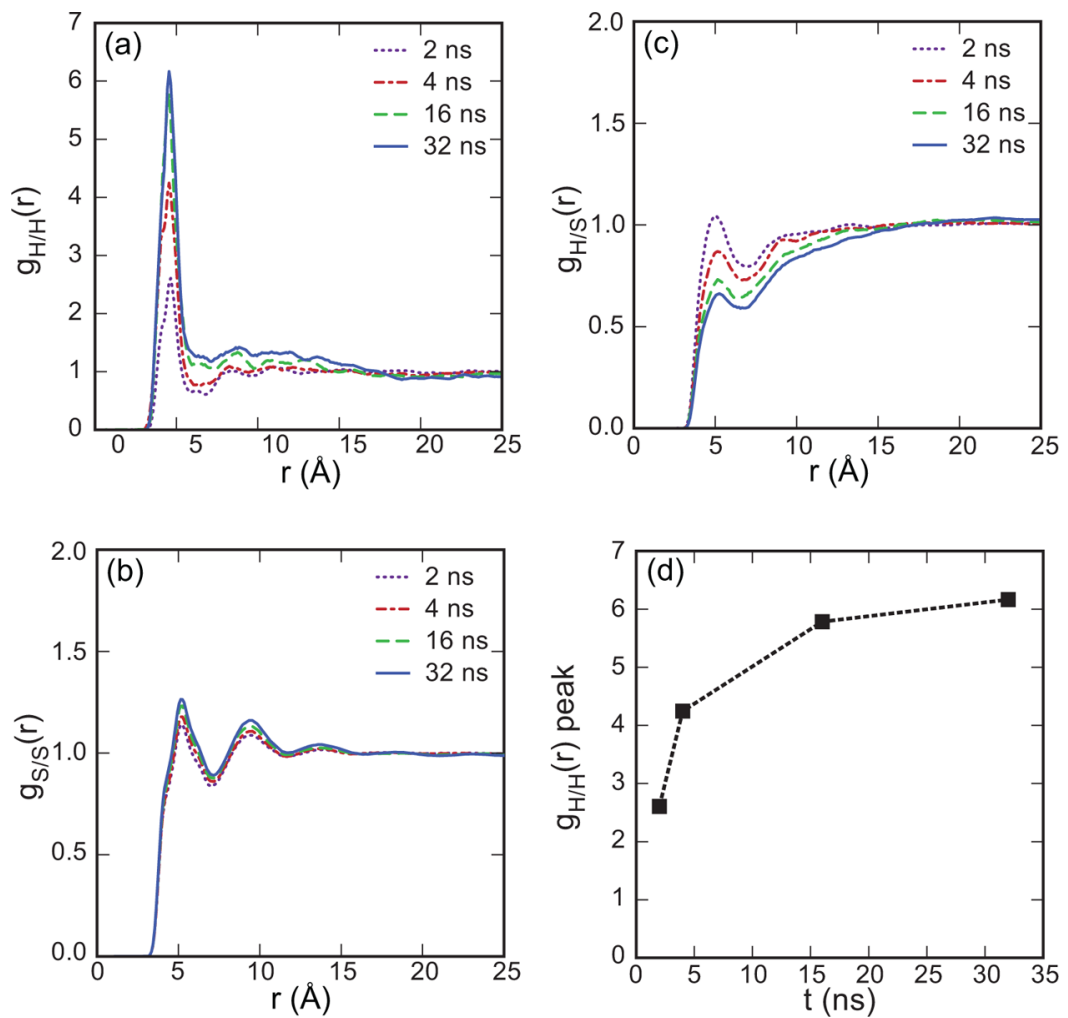

Figure 2. Evolution of radial distribution functions (a) $g_{\mathrm{H} / \mathrm{H}}(r),(\mathrm{b}) g_{\mathrm{H} / \mathrm{S}}(r)$, and (c) $g_{\mathrm{S} / \mathrm{S}}(r)$, with equilibration time, and (d) convergence of the $g_{\mathrm{H} / \mathrm{H}}(r)$ peak.

Iterative Boltzmann Inversion. We use the distributions $P_{\mathrm{H}-\mathrm{H}}(l), P_{\mathrm{H}-\mathrm{S}}(l)$, and $P_{\mathrm{S}-\mathrm{S}}(l)$ measured from atomistic simulations to derive the CG bond-stretching potentials $\mathcal{V}_{\mathrm{H}-\mathrm{H}}^{\mathrm{str}}(l), \mathcal{V}_{\mathrm{H}-\mathrm{S}}^{\mathrm{str}}(l)$, and $\mathcal{V}_{S-S}^{\text {str }}(l)$ via Boltzmann inversion:

$$
\mathcal{V}_{x}^{\mathrm{str}}(l)=-k_{\mathrm{B}} T \ln \left[P_{x}(l) / l^{2}\right], \quad x \equiv \mathrm{H}-\mathrm{H}, \mathrm{H}-\mathrm{S}, \mathrm{S}-\mathrm{S}
$$

where $k_{B}$ is the Boltzmann constant and the $l^{2}$ term accounts for the degeneracy in the position of a bead located at a fixed distance $l$ from another bead. ${ }^{31}$ The potentials derived in this manner typically have a quadratic shape about their minimum, implying a harmonic underlying potential. Therefore, one can simply model the bond-stretching potential as a harmonic spring:

$$
\mathcal{V}_{x}^{\mathrm{str}}(l) \approx \frac{k_{x}}{2}\left(l-l_{x}\right)^{2}, \quad x \equiv \mathrm{H}-\mathrm{H}, \mathrm{H}-\mathrm{S}, \mathrm{S}-\mathrm{S}
$$

where the effective bond stiffnesses $k_{\mathrm{H}-\mathrm{H}}, k_{\mathrm{H}-\mathrm{S}}$, and $k_{\mathrm{S}-\mathrm{S}}$ and equilibrium bond lengths $l_{\mathrm{H}-\mathrm{H}}, l_{\mathrm{H}-\mathrm{S}}$, and $l_{\mathrm{S}-\mathrm{S}}$ for the three types of bonds can be obtained fitting the potentials derived via eq 5 to the harmonic functions described in eq 6 .

Similarly, one can directly derive the CG angle-bending potentials $\mathcal{V}_{\mathrm{H}-\mathrm{H}-\mathrm{S}}^{\mathrm{bend}}(\theta), \mathcal{V}_{\mathrm{H}-\mathrm{S}-\mathrm{S}}^{\mathrm{bend}}(\theta)$, and $\mathcal{V}_{\mathrm{S}-\mathrm{S}-\mathrm{S}}^{\text {bend }}(\theta)$ via Boltzmann inversion of the bending angle distributions $P_{\mathrm{H}-\mathrm{H}-\mathrm{S}}(\theta)$, and $P_{\mathrm{S}-\mathrm{S}-\mathrm{S}}(\theta)$ measured from atomistic simulations:

$$
\begin{gathered}
\mathcal{V}_{x}^{\text {bend }}(\theta)=-k_{\mathrm{B}} T \ln \left[P_{x}(\theta) / \sin \theta\right], \\
x \equiv \mathrm{H}-\mathrm{H}-\mathrm{S}, \mathrm{H}-\mathrm{S}-\mathrm{S}, \mathrm{S}-\mathrm{S}-\mathrm{S}
\end{gathered}
$$

where the $\sin \theta$ term accounts for the degeneracy in the position of a bead subtending an angle $\theta$ with a bond. As in the case of bond lengths, the bending potential in eq 7 can be approximated as a harmonic angular spring:

$$
\mathcal{V}_{x}^{\text {bend }}(\theta) \approx \frac{k_{x}}{2}\left(\theta-\theta_{x}\right)^{2}, \quad x \equiv \mathrm{H}-\mathrm{H}-\mathrm{S}, \mathrm{H}-\mathrm{S}-\mathrm{S}, \mathrm{S}-\mathrm{S}-\mathrm{S}
$$

where the effective bending angle stiffnesses $k_{\mathrm{H}-\mathrm{H}-\mathrm{S}}, k_{\mathrm{H}-\mathrm{S}-\mathrm{S}}$, and $k_{\mathrm{S}-\mathrm{S}-\mathrm{S}}$ and equilibrium bending angles $\theta_{\mathrm{H}-\mathrm{H}-\mathrm{S}}, \theta_{\mathrm{H}-\mathrm{S}-\mathrm{S}}$, and $\theta_{\mathrm{S}-\mathrm{S}-\mathrm{S}}$ for the three types of bending angles can be obtained fitting the potentials derived via eq 7 to the functions described in eq 8 .

The bond stretching and bending potentials derived from such direct Boltzmann inversion of bond length and angle distributions are usually good approximations of the true underlying potentials. The reason is that each distribution has a stiff, localized dependence on its corresponding order parameter (bond length or angle) and is therefore uncorrelated with other distributions. However, this is not true for nonbonded RDFs, which arise from softer potentials and include significant contributions from beads other than the pair of beads used in the RDF calculation (multibody effects), especially in condensed phases like polymer melts. Hence, direct Boltzmann inversion of the RDFs will not generally yield the correct nonbonded pair potentials.

To derive the true CG nonbonded pair potentials, we perform $\mathrm{MD}$ simulations of CG $\mathrm{H}_{2} \mathrm{~S}_{14}$ bead-chains at the same relevant densities and temperatures as the corresponding atomistic simulations. For the CG force field, we use initial guesses of the three CG nonbonded potentials obtained via Boltzmann inversion:

$$
\mathcal{V}_{x, 0}^{\mathrm{nonb}}(r)=-k_{\mathrm{B}} T \ln g_{x}(r), \quad x \equiv \mathrm{H} / \mathrm{H}, \mathrm{H} / \mathrm{S}, \mathrm{S} / \mathrm{S}
$$

along with the set of six bond-stretching and bending potentials obtained via eqs $5-8$. These CG MD simulations yield RDFs $g_{x, 0}(r)$ that may differ from the target RDFs $g_{x}(r)$ obtained from atomistic simulations, where $x \equiv \mathrm{H} / \mathrm{H}, \mathrm{H} / \mathrm{S}$, or $\mathrm{S} / \mathrm{S}$. On the basis of differences between the two sets of RDFs, the CG nonbonded potentials $\mathcal{V}_{x, 0}^{\text {nonb }}(r)$ can be iteratively improved by the following correction:

$$
\begin{aligned}
& \mathcal{V}_{x, i+1}^{\mathrm{nonb}}(r)=\mathcal{V}_{x, i}^{\mathrm{nonb}}(r)-\alpha k_{\mathrm{B}} T \ln \left(\frac{g_{x, i}(r)}{g_{x}(r)}\right), \\
& x \equiv \mathrm{H} / \mathrm{H}, \mathrm{H} / \mathrm{S}, \mathrm{S} / \mathrm{S}
\end{aligned}
$$

where $i$ stands for the iteration number and $\alpha<1$ is a scaling factor that helps improve the convergence and stability of the IBI process. 
In general, the value of $\alpha$ is problem specific, however for the polyurea model, we find that a value of $\alpha=0.05$ is sufficient for robust optimization of the potentials. This iterative procedure is continued until all nonbonded potentials converge to their limiting profiles, as denoted by $\mathcal{V}_{\mathrm{H} / \mathrm{H}}^{\mathrm{nonb}}(r), \mathcal{V}_{\mathrm{H} / \mathrm{S}}^{\mathrm{nonb}}(r)$, and $\mathcal{V}_{\mathrm{S} / \mathrm{S}}^{\mathrm{nonb}}(r)$. At this point, the CG simulations should yield the same RDFs as the targets $g_{\mathrm{H} / \mathrm{H}}(r), g_{\mathrm{H} / \mathrm{S}}(r)$, and $g_{\mathrm{S} / \mathrm{S}}(r)$ obtained from the atomistic $\mathrm{MD}$ simulations. Even after matching the target RDFs, the pressures computed from the CG simulations might not match the target pressure. To resolve this potential issue, we modify the pressure by adding a linear term to the CG nonbonded pair potential after each iteration until we get the correct pressure: ${ }^{15,32-34}$

$$
\Delta \mathcal{V}_{\mathrm{pc}}(r)=A k_{\mathrm{B}} T\left(1-\frac{r}{r_{\text {cut }}}\right)
$$

where the value of $A$ depends on the deviation from the target pressure.

Figure 3 summarizes our implementation of the IBI method. We begin by placing $150 \mathrm{H}_{2} \mathrm{~S}_{14} \mathrm{CG}$ bead-chains in random, nonoverlapped

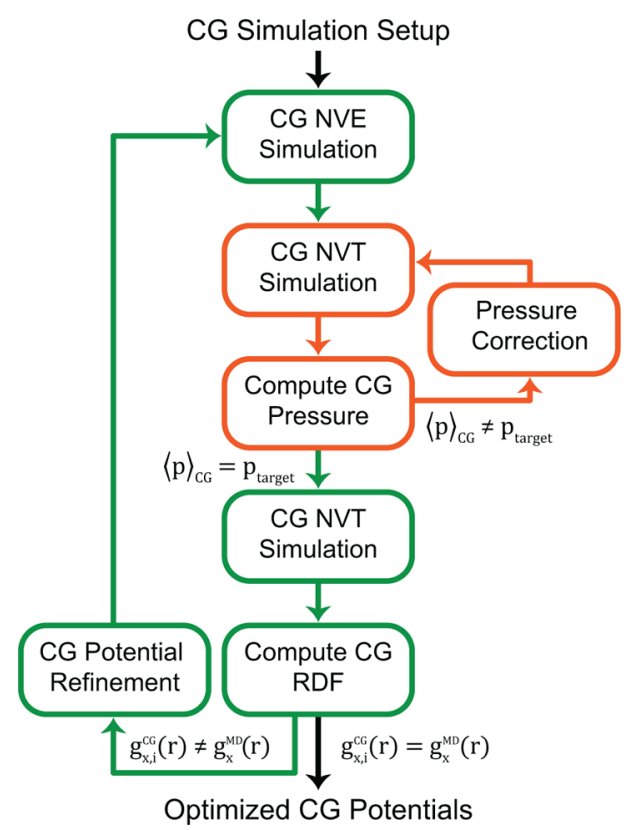

Figure 3. IBI implementation procedure.

configurations within a cubic simulation box of size commensurate with the target density. The initial bond lengths and angles of the chains are set equal to their respective equilibrium values obtained from eqs 6 and 8. Each IBI iteration step starts with a 0.8 ns equilibration step at $300 \mathrm{~K}$. For the force field, we utilize the CG bondstretching and bending potentials derived from atomistic simulations, eqs 6 and 8, along with the CG nonbonded potentials obtained from the previous iteration step, except for the first step where we use the direct Boltzmann inversion of the atomistic RDFs as an initial guess for the nonbonded potentials. The equilibration step is followed by a cycle of pressure-correction steps. During this cycle, the CG system is held at $T=300 \mathrm{~K}$ in an NVT ensemble to compute a ensemble- and timeaveraged pressure. This pressure is compared against the target pressure $(p=1 \mathrm{~atm})$ so as to adjust the CG nonbonded potential according to eq 11 . The pressure correction cycle is repeated until the computed CG pressure matches the target pressure, which is quite rapid and typically converges within 3-5 iterations. At this point, longer CG MD simulations (1.6 ns) are performed in the NVT ensemble at $T=300 \mathrm{~K}$, using the pressure-corrected nonbonded potentials to compute the RDFs. These CG RDFs are compared against the atomistic RDFs to propose more accurate estimates of $\mathrm{CG}$ nonbonded potentials via eq 10 . We iterate the IBI steps discussed above, inclusive of the pressure correction cycle, until the CG RDFs converge to the atomistic RDFs. As with the atomistic simulations, all CG MD simulation steps are performed using the LAMMPS software. ${ }^{35}$

Model Validation. The utility of a CG model is measured by its ability to correctly predict structural and thermodynamic properties of the atomistic system that did not feature in the CG force field parametrization. We validate our CG model by comparing several of its properties, including the density, heat capacity, and stress relaxation spectrum against those computed from atomistic simulations. In particular, we use four different monodisperse, short-chain systems in which the length of the polyurea chains varies from one to eight blocks $-\mathrm{H}_{2} \mathrm{~S}_{14},\left(\mathrm{H}_{2} \mathrm{~S}_{14}\right)_{3},\left(\mathrm{H}_{2} \mathrm{~S}_{14}\right)_{5}$, and $\left(\mathrm{H}_{2} \mathrm{~S}_{14}\right)_{8}$. We also examine how well the CG model can predict properties of polyurea melts containing much longer chains, $\left(\mathrm{H}_{2} \mathrm{~S}_{14}\right)_{20}$.

Heat Capacity. The specific heat capacity $C_{\mathrm{v}}$ plays an important role in energy storage and dissipation. We compute the specific heat $C_{\mathrm{v}}$ of our polyurea systems from the fluctuation in the total energy of the system in the NVT ensemble: ${ }^{36}$

$$
C_{V}=\left.\frac{\partial\langle E\rangle}{\partial T}\right|_{V}=\frac{\left\langle\delta E^{2}\right\rangle}{k_{\mathrm{B}} T^{2}}
$$

where $\langle E\rangle$ and $\left\langle\delta E^{2}\right\rangle$ are the mean value and variance of the total energy, respectively. Following Zhou et al. ${ }^{37}$ we decompose the specific heat in terms of the kinetic and potential energy contributions as

$$
C_{V}=\left.\frac{\partial\langle K\rangle}{\partial T}\right|_{V}+\left.\frac{\partial\langle U\rangle}{\partial T}\right|_{V}
$$

From the equipartition theorem, $\langle K\rangle=(3 / 2) N k_{B} T$, and therefore

$$
\frac{\partial\langle K\rangle}{\partial T}=\frac{3}{2} N k_{\mathrm{B}}
$$

where $N$ represents the number of atoms or CG beads in the system. This term represents the ideal heat capacity of the system. The remainder of the heat capacity, due to the interaction between particles, can be formulated assuming that the potential energy follows the Boltzmann distribution:

$$
\left.\frac{\partial\langle U\rangle}{\partial T}\right|_{V}=\frac{\left\langle\delta U^{2}\right\rangle}{k_{\mathrm{B}} T^{2}}+\frac{\langle\delta U \delta K\rangle}{k_{\mathrm{B}} T^{2}}
$$

where $\langle\delta U \delta K\rangle$ is the ensemble-averaged cross-correlation of the variances of potential energy and kinetic energy.

Diffusion Coefficient. The self-diffusion coefficient $D_{\mathrm{s}}$ characterizes the mobility of the polymer chains within the melt. We compute $D_{s}$ of the center of mass of polyurea chains, both CG and atomistic, from the slope of the mean square displacement: ${ }^{31}$

$$
D_{\mathrm{s}}=\lim _{t \rightarrow \infty} \frac{1}{6 t}\left\langle\mathbf{r}(t)-\left.\mathbf{r}(0)\right|^{2}\right\rangle
$$

where $t$ denotes time, $\mathbf{r}(t)$ is the position of the center of mass of the polymer chain $i$ at time $t$, and $\langle\cdots\rangle$ denotes ensemble average of the quantity within the angular brackets over all chains in the simulation and reference positions $\mathbf{r}(0)$.

Stress Relaxation. The stress relaxation spectrum $G(t)$ is perhaps the most important quantity relevant to energy dissipation. We compute $G(t)$ from the stress autocorrelation function: ${ }^{9}$

$$
G(t)=\frac{V}{k_{\mathrm{B}} T}\left\langle\sigma_{x y}(t) \sigma_{x y}(0)\right\rangle
$$

Here $\sigma_{x y}$ is the instantaneous shear stress computed from the virial theorem

$$
\sigma_{x y}=\frac{1}{V}\left[\sum_{i=1}^{N} m_{i} v_{i x} v_{i y}+\sum_{i=1}^{N-1} \sum_{j=i+1}^{N} r_{i j x} F_{i j y}\right]
$$

where $V$ is the volume of the simulation box; $m_{i}, v_{i x}$ and $v_{i y}$ are the mass and $x$ - and $y$-component velocities of bead $i$ and $j$, respectively; 

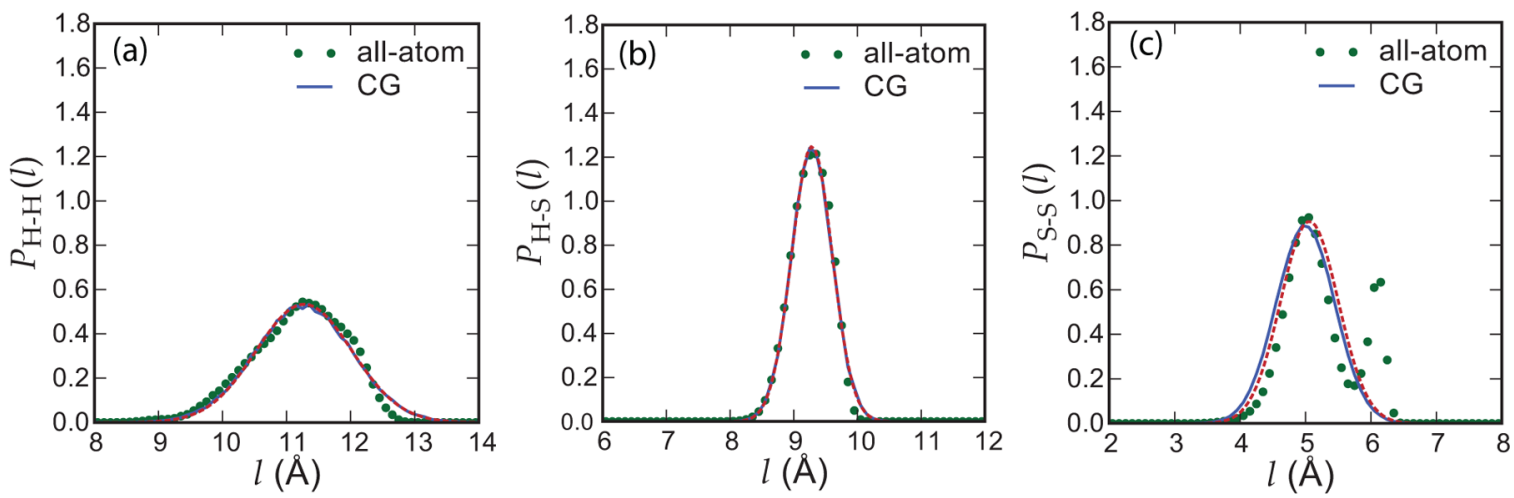

Figure 4. Bond length distributions (a) $P_{\mathrm{H}-\mathrm{H}}(l),(\mathrm{b}) P_{\mathrm{H}-\mathrm{S}}(l)$, and $(\mathrm{c}) P_{\mathrm{S}-\mathrm{S}}(l)$ obtained from atomistic MD simulations and from CG MD simulation after IBI convergence. The dashed line shows a weighted Gaussian fit to the bond distributions obtained from atomistic MD simulations.
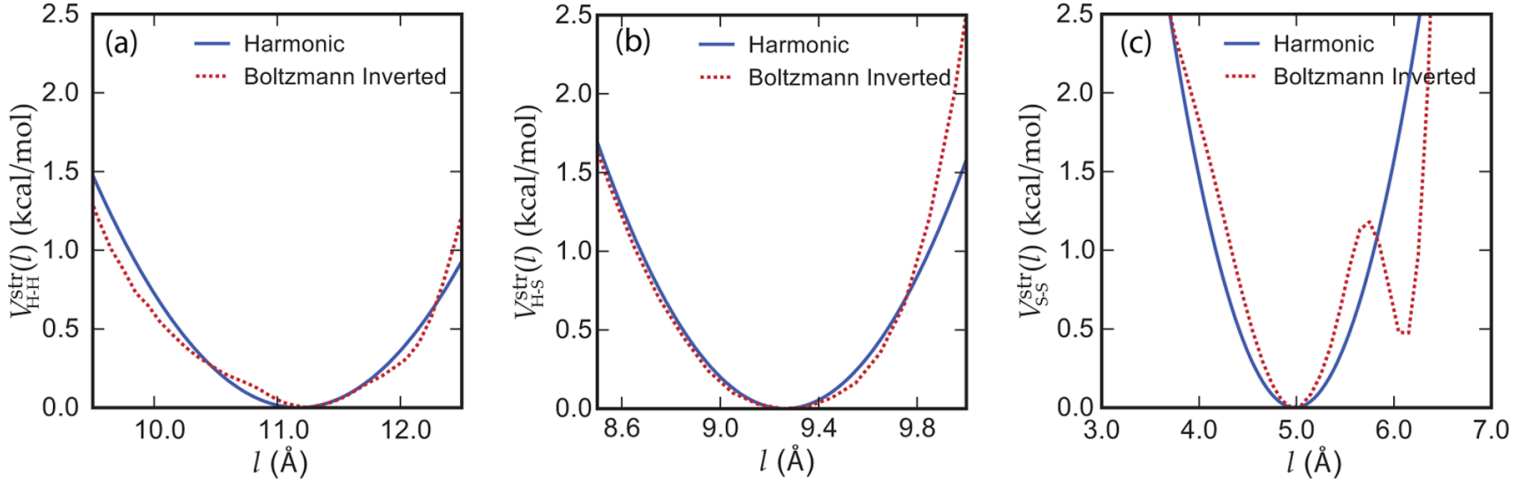

Figure 5. Bond-stretching potentials $(\mathrm{a}) \mathcal{V}_{\mathrm{H}-\mathrm{H}}^{\mathrm{str}}(l),(\mathrm{b}) \mathcal{V}_{\mathrm{H}-\mathrm{S}}^{\mathrm{str}}(l)$, and $(c) \mathcal{V}_{\mathrm{S}-\mathrm{S}}^{\text {str }}(l)$ obtained from Boltzmann inversion of bond length distributions and from harmonic approximation based on fitted parameters summarized in Table 1.

and $r_{i j x}$ and $F_{i j y}$ are the $x$-component separation distance and $y$ component force acting between beads $i$ and $j$, respectively. Since the stresses $\sigma_{x y}, \sigma_{x z}$ and $\sigma_{y z}$ are equivalent for an isotropic system, we use the average of the autocorrelations functions obtained from the three stresses to obtain smoother estimates of $G(t)$.

Simulation Details. The above properties are computed for both the atomistic and CG systems using equilibrium MD simulations. For the atomistic simulations, the number of chains in each system are varied to keep the net number of $\mathrm{H}_{2} \mathrm{~S}_{14}$ blocks fixed at 40 , so that each system contains about 10,000 atoms. The simulations are performed with a time step of $1 \mathrm{fs}$. The fully atomistic systems are constructed in a random, nonoverlapping configurations and annealed for $8 \mathrm{~ns}$ in the NPT ensemble at $T=500 \mathrm{~K}$ and $p=1 \mathrm{~atm}$, followed by a decrease in temperature to $300 \mathrm{~K}$ for $8 \mathrm{~ns}$, and equilibrated at $300 \mathrm{~K}$ for $16 \mathrm{~ns}$. Thereafter, the equilibrated systems are simulated in the NVT ensemble at $300 \mathrm{~K}$ for $100 \mathrm{~ns}$. The MD simulations of the corresponding CG chains are equilibrated in the NPT ensemble at $300 \mathrm{~K}$ for $250 \mathrm{~ns}$ because of the limited temperature transferability of CG potentials, however this simpler equilibration is sufficient due to their faster dynamics. The number of polyurea blocks is also increased to 500 for the CG systems and the integration time step is increased to $25 \mathrm{fs}$. For each simulation, the densities, heat capacities, and stress relaxation profiles are computed from the atomic/CG bead positions and velocities to test how well the CG model reproduced these properties in comparison to the corresponding fully atomistic MD simulations.

\section{RESULTS}

Model Parameterization. As discussed in the previous section, nine different structural distributions are computed from atomistic $\mathrm{MD}$ simulations of short-chain polyurea systems $\left(\mathrm{H}_{2} \mathrm{~S}_{14}\right)$ in the melt state at atmospheric conditions. Figure 4 presents the bond length distributions $P_{\mathrm{H}-\mathrm{H}}(l), P_{\mathrm{H}-\mathrm{S}}(l)$, and $P_{S-S}(l)$ computed from these simulations. All three distributions exhibit a typical Gaussian shape, suggesting that the underlying bond-stretching potentials can indeed be modeled as harmonic springs. Figure 5 shows the Boltzmanninverted estimates of the underlying bond-stretching potential $\mathcal{V}_{\mathrm{H}-\mathrm{H}}^{\text {str }}(l), \mathcal{V}_{\mathrm{H}-\mathrm{S}}^{\mathrm{str}}(l)$, and $\mathcal{V}_{\mathrm{S}-\mathrm{S}}^{\mathrm{str}}(l)$ obtained via eq 5 . Also shown in the same plots are the excellent fits to these potentials using the harmonic equation presented in eq 6 , with the exception of the $S-S$ bond length distribution that has a minor second peak due to two torsional conformations. While the harmonic bond approximation does not allow the CG model to reproduce this double-peaked bond length distribution, it has a negligible effect on the structural and thermodynamic properties of the CG system. The resulting fits yield the effective bond stiffnesses and equilibrium bond lengths tabulated in Table 1 . These values

Table 1. Summary of Bond and Angle Parameters for CG Model of Polyurea

\begin{tabular}{ccc} 
bond type & bond length $(\AA)$ & stiffness $\left(\mathrm{kcal} / \mathrm{mol} / \AA^{2}\right)$ \\
H-H & 11.17 & 1.05 \\
H-S & 9.26 & 5.82 \\
S-S & 4.98 & 3.03 \\
angle type & angle $(\mathrm{deg})$ & stiffness $\left(\mathrm{kcal} / \mathrm{mol} / \mathrm{rad}^{2}\right)$ \\
H-H-S & 128.4 & 1.60 \\
H-S-S & 134.5 & 0.76 \\
S-S-S & 169.5 & 0.64 \\
\hline
\end{tabular}

indicate that the $S-S$ bonds are the shortest while the $H-S$ bonds are the stiffest, while the $H-H$ bonds are the longest and softest, clearly due to the smaller size of the $S$ segments. 
Figure 6 shows the bending angle distributions $P_{\mathrm{H}-\mathrm{H}-\mathrm{S}}(\theta)$, $P_{\mathrm{H}-\mathrm{S}-\mathrm{S}}(\theta)$, and $P_{\mathrm{S}-\mathrm{S}-\mathrm{S}}(\theta)$ measured from atomistic simulations. Boltzmann-inverting the distributions via eq 8 yields the effective bending potentials $\mathcal{V}_{\mathrm{H}-\mathrm{H}-\mathrm{S}}^{\text {bend }}(\theta), \mathcal{V}_{\mathrm{H}-\mathrm{S}-\mathrm{S}}^{\text {bend }}(\theta)$, and $\mathcal{V}_{\mathrm{S}-\mathrm{S}-\mathrm{S}}^{\text {bend }}(\theta)$, which are plotted in Figure 7. The potentials exhibit a harmonic shape, though somewhat less harmonic than the bond stretching potentials. Nonetheless, fitting the potentials to the harmonic function in eq 8 yields the parameters tabulated in Table 1 .

Figure 8 shows the RDFs $g_{\mathrm{H} / \mathrm{H}}(r), g_{\mathrm{H} / \mathrm{S}}(r)$, and $g_{\mathrm{S} / \mathrm{S}}(r)$ corresponding to the three types of nonbonded pairwise interactions, as computed from the atomistic MD simulations. All three RDFs exhibit a discernible peak at short separation distances.
As expected, $g_{\mathrm{H} / \mathrm{H}}(r)$ displays the highest peak, indicating strong aggregation of hard segments into hard domains due to $\pi$-stacking of the aromatic rings and hydrogen bonding between the urea linkages; in contrast, $g_{\mathrm{S} / \mathrm{S}}(r)$ and $g_{\mathrm{H} / \mathrm{S}}(r)$ display much smaller peaks, suggesting weaker interactions between soft segments and between soft and hard segments, respectively. We also observe some modulations in the RDFs beyond these first peaks, implying higher-order caging effects. However, these modulations decay quickly, and by $r \geq 22 \AA$ for $g_{\mathrm{H} / \mathrm{H}}(r)$ and $r \geq 16 \AA$ for both $g_{\mathrm{H} / \mathrm{S}}(r)$ and $g_{\mathrm{S} / \mathrm{S}}(r)$, plateau to their nominal bulk value of 1 .

To derive the CG nonbonded potentials $\mathcal{V}_{\mathrm{H} / \mathrm{H}}^{\mathrm{nonb}}(r), \mathcal{V}_{\mathrm{H} / \mathrm{S}}^{\mathrm{nonb}}(r)$, and $\mathcal{V}_{\mathrm{S} / \mathrm{S}}^{\mathrm{nonb}}(r)$, we use the above RDFs to obtain initial guesses
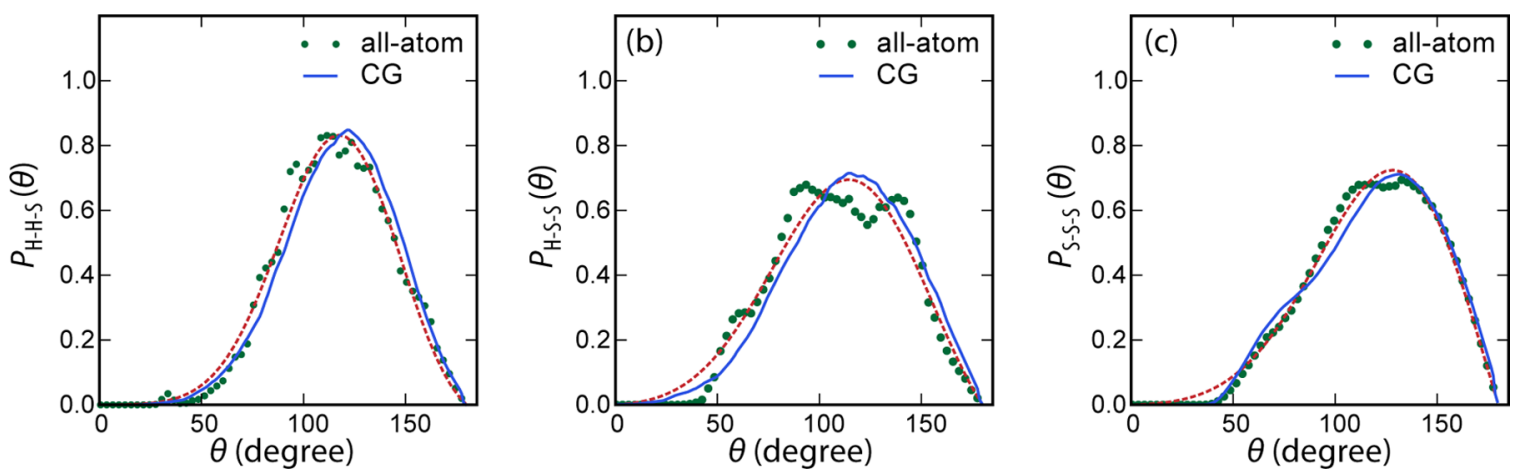

Figure 6. Bending angle distributions (a) $P_{\mathrm{H}-\mathrm{H}-\mathrm{S}}(\theta),(\mathrm{b}) P_{\mathrm{H}-\mathrm{S}-\mathrm{S}}(\theta)$, and (c) $P_{\mathrm{S}-\mathrm{S}-\mathrm{S}}(\theta)$ obtained from all-atom MD simulations and from CG MD simulations after IBI convergence. The dashed line shows a weighted Gaussian fit to the all-atom angle distributions via eqs 7 and 8 .
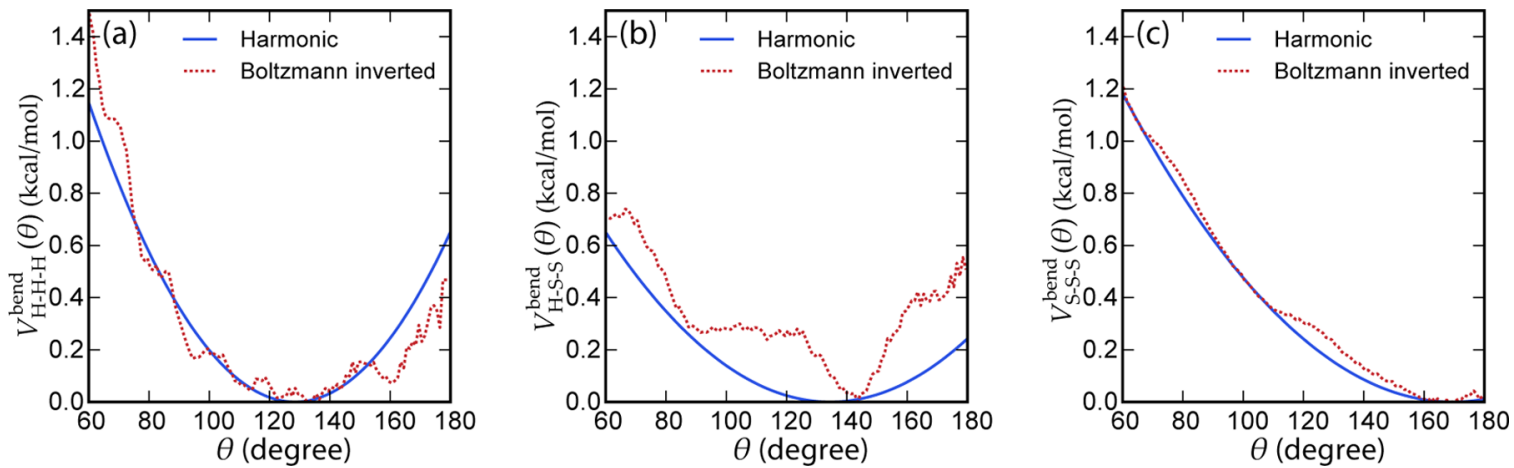

Figure 7. Bending angle potentials $(a) \mathcal{V}_{\mathrm{H}-\mathrm{H}-\mathrm{S}}^{\text {bend }}(\theta),(b) \mathcal{V}_{\mathrm{H}-\mathrm{S}-\mathrm{S}}^{\text {bend }}(\theta)$, and $(c) \mathcal{V}_{\mathrm{S}-\mathrm{S}-\mathrm{S}}^{\text {bend }}(\theta)$ obtained from Boltzmann inversion of bending angle distributions and from harmonic approximation based on the fitted parameters summarized in Table 1 . The Boltzmann inverted potential is shifted by an arbitrary constant so that its minimum value is zero energy. Note that this shift plays no role in determining the structure and dynamics of the CG system.
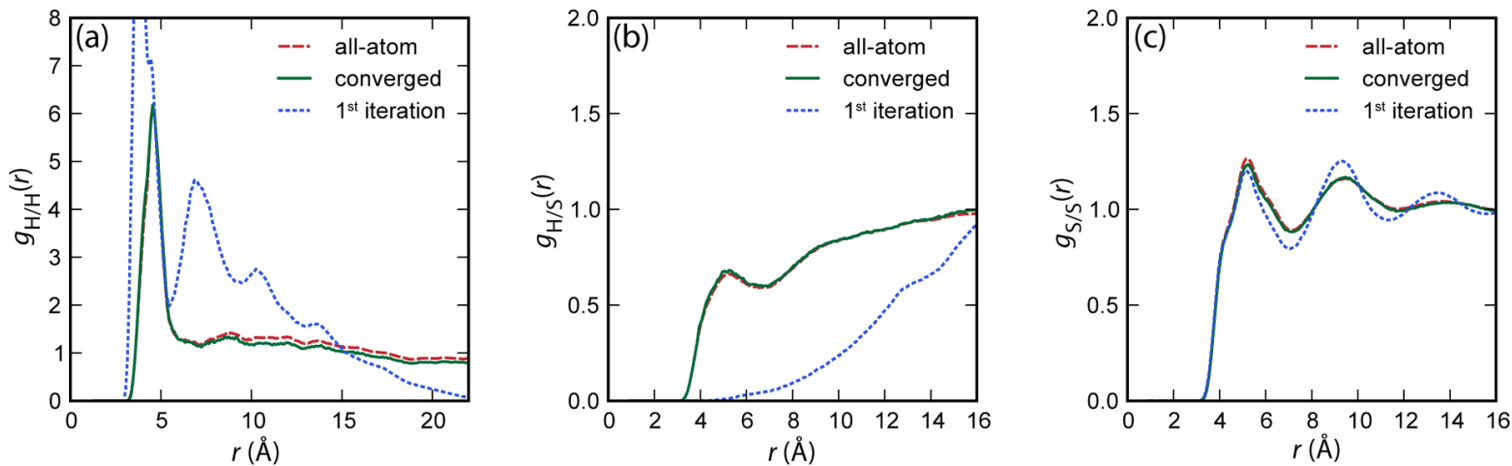

Figure 8. Radial distribution functions (a) $g_{\mathrm{H} / \mathrm{H}}(r),(\mathrm{b}) g_{\mathrm{H} / \mathrm{S}}(r)$, and $(\mathrm{c}) g_{\mathrm{S} / \mathrm{S}}(r)$ obtained from all-atom MD simulations, CG MD simulations after IBI convergence and CG MD simulations during the first IBI iteration, i.e., based on the initial guess potential obtained from Boltzmann inversion the all-atom MD RDFs. 

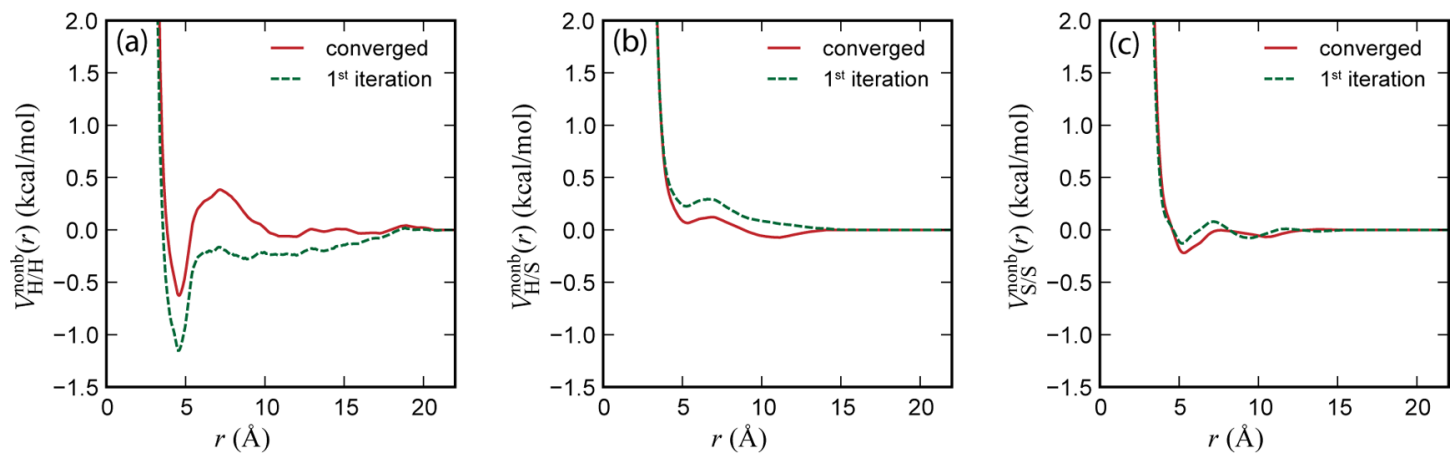

Figure 9. Nonbonded potentials (a) $\mathcal{V}_{\mathrm{H} / \mathrm{H}}^{\mathrm{nonb}}(r),(\mathrm{b}) \mathcal{V}_{\mathrm{H} / \mathrm{S}}^{\mathrm{nonb}}(r)$, and (c) $\mathcal{V}_{\mathrm{S} / \mathrm{S}}^{\mathrm{nonb}}(r)$. obtained from convergence of the IBI method compared against those used in the first IBI iteration (i.e., obtained from Boltzmann inversion of the all-atom RDFs).

of the nonbonded potentials via eq 10 . We then use these guesses along with the bond-stretching and bending potentials derived earlier to initiate the IBI procedure. In Figure 8 we compare the RDFs computed from CG MD simulations $g_{\mathrm{H} / \mathrm{H}, i}$ $g_{\mathrm{H} / \mathrm{S}, i}$, and $g_{\mathrm{S} / \mathrm{S}, i}$ after the first IBI iteration $(i=1)$, and after convergence against the target RDFs obtained from atomistic simulations. It can be observed that the IBI procedure implemented here leads to converged RDFs for all three types of nonbonded interactions within 80 iterations. We also note that among the three RDFs, those corresponding to $S / S$ interactions converge the fastest (within 10 iterations) while those corresponding to $\mathrm{H} / \mathrm{H}$ interactions converge the slowest because of the phase segregation between hard and soft segments of polyurea.

The final, converged CG nonbonded potentials $\mathcal{V}_{\mathrm{H} / \mathrm{H}}^{\mathrm{nonb}}(r)$, $\mathcal{V}_{\mathrm{H} / \mathrm{S}}^{\mathrm{nonb}}(r)$, and $\mathcal{V}_{\mathrm{S} / \mathrm{S}}^{\text {nonb }}(r)$ are plotted in Figure 9 . We observe that $\mathcal{V}_{\mathrm{H} / \mathrm{H}}^{\mathrm{nonb}}(r)$ exhibits a much deeper energy minimum $\left(\sim 1 k_{B} T\right)$ than the other two nonbonded potentials $\left(\ll 1 k_{B} T\right)$. Clearly, it is this difference in the interactions of hard and soft segments that is responsible for the microphase separation observed in polyurea systems. We have also plotted in the same figure the initial guesses for the nonbonded potentials, as obtained from Boltzmann inversion. The converged nonbonded potentials are clearly very different from the initial guesses, which emphasizes the importance of the IBI procedure in deriving correct nonbonded interaction potentials. These differences also aptly illustrate the distinction between a pairwise potential energy, as captured by the converged potentials $\mathcal{V}_{x}^{\text {nonb }}(r)$, and a potential of mean force (or free energy), as captured by the initial guesses $\mathcal{V}_{x, 0}^{\text {nonb }}(r)$.

Our current IBI procedure of simultaneously optimizing the CG nonbonded potentials $\mathcal{V}_{\mathrm{H} / \mathrm{H}}^{\mathrm{nonb}}(r), \mathcal{V}_{\mathrm{H} / \mathrm{S}}^{\mathrm{nonb}}(r)$, and $\mathcal{V}_{\mathrm{S} / \mathrm{S}}^{\mathrm{nonb}}(r)$ seems to work efficiently and robustly. We also attempted a slightly different scheme for deriving the CG nonbonded potentials involving sequential optimization of the potentials. In this sequential scheme, we first derived $\mathcal{V}_{\mathrm{S} / \mathrm{S}}^{\mathrm{nonb}}(r)$ by implementing IBI on $S_{14}$ chains, using the bonded potentials $\mathcal{V}_{\mathrm{S}-\mathrm{S}}^{\text {str }}(r)$ and $\mathcal{V}_{\mathrm{S}-\mathrm{S}-\mathrm{S}}^{\text {bend }}(r)$ derived earlier. Next, we derived $\mathcal{V}_{\mathrm{H} / \mathrm{H}}^{\mathrm{nonb}}(r)$ by implementing IBI on $\mathrm{H}_{2}$ dimers, using the bonded potential $\mathcal{V}_{\mathrm{H}-\mathrm{H}}^{\mathrm{str}}(r)$ derived earlier. Finally, $\mathcal{V}_{\mathrm{H} / \mathrm{S}}^{\text {nonb }}(r)$ was derived by implementing IBI on $\mathrm{H}_{2} \mathrm{~S}_{14}$ chains using the two nonbonded CG potentials and six bonded CG potentials derived earlier. We find that while this scheme yields similar estimates of
$\mathcal{V}_{\mathrm{S} / \mathrm{S}}^{\mathrm{nonb}}(r)$ as those obtained from simultaneous IBI optimization, the IBI step for deriving $\mathcal{V}_{\mathrm{H} / \mathrm{H}}^{\mathrm{nonb}}(r)$, and $\mathcal{V}_{\mathrm{H} / \mathrm{S}}^{\mathrm{nonb}}(r)$ was generally unstable and failed to converge because sequential optimization is poorly suited for CG models of polymers that have a large contrast in affinities between the different bead types, as is the case with our CG model of polyurea. Thus, we suggest that simultaneous IBI optimization, as opposed to sequential optimization, is perhaps the most efficient strategy for deriving CG nonbonded potentials of heterogeneous systems composed of more than one type of bead.

Model Validation. To validate the CG model of polyurea developed above, we have computed several thermophysical and structural properties of short-chain polyurea systems using the CG model and compared the properties against those computed from fully atomistic models. As mentioned earlier, we carry out this comparison for four different systems, each containing polyurea chains of different lengths: $\left(\mathrm{H}_{2} \mathrm{~S}_{14}\right)_{n}$, where $n=1,3,5$ and 8 . Because of the computational expense in generating equilibrium configurations with all-atom models, only the 1-block polyurea end-to-end distribution, morphology, stress relaxation spectra, and dynamic scaling factor are computed as verification of the CG model.

We begin by comparing the average densities $\rho$ and constantvolume heat capacities $C_{\mathrm{v}}$ computed from the CG and atomistic models for the four different polyurea systems, which are plotted in Figure 10 as a function of chain length. Each atomistic
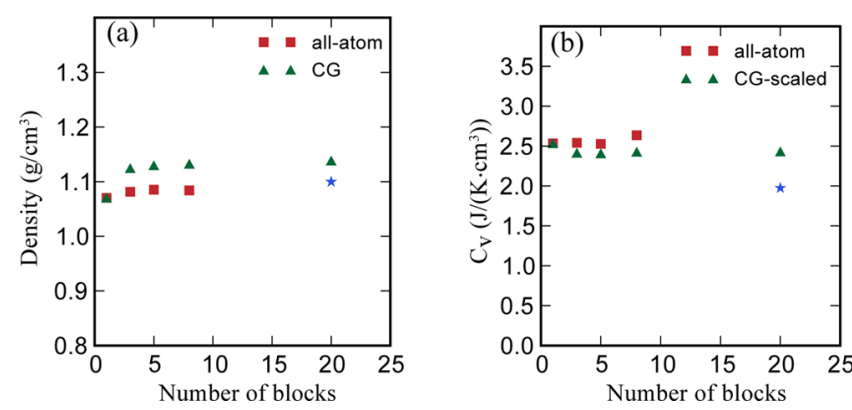

Figure 10. Comparison of (a) density and (b) heat capacity between all-atom MD simulations and CG simulations for oligomeric polyurea of varying chain lengths. Experimental values are denoted with asterisks.

model was simulated with a single system due to the large computational expense to equilibrate all-atom systems. For the CG models, five different replica systems were simulated and the results averaged to produce the predicted density and heat capacity values. The standard deviation of the density 
predictions was less than $0.1 \%$, while for the heat capacity, the standard deviation was less than $1 \%$.

We observe an increase in density with increasing chain length for both the CG and atomistic systems, although the CG models exhibit a larger increase, particularly at the transition from one to two block chains. The largest difference in the predicted density is seen for the 8 block system, where the disparity is $4.4 \%$. The density of polyurea reported by Amini et al. ${ }^{38}$ is $1.1 \mathrm{~g} / \mathrm{cm}^{3}$ for a molecular weight consistent with a 20 block chain is plotted with an asterisk as a reference.

Comparison of the computed heat capacity between atomistic and CG models via eqs 14 and 15 is less straightforward as the thermodynamic temperature of the system explicitly depends on the number of degrees of freedom in the system. As the CG models contain significantly fewer degrees of freedom per unit volume, their computed heat capacities are proportionally lower. A single block $\left(\mathrm{H}_{2} \mathrm{~S}_{14}\right)$ of polyurea contains 246 atoms or $16 \mathrm{CG}$ beads with the previously described mapping, leading to a scaling factor of $C_{k}=15.375$ for the ideal heat capacity of the system. For the nonideal components, we compare the ratio of the fluctuation terms $\left\langle\delta U^{2}\right\rangle$ and $\langle\delta U \delta K\rangle$ between the CG and all-atom MD simulations of single block systems to empirically determine scaling factors of $C_{u}=9.30$, and $C_{u k}=26.67$, respectively, with the assumption that the partitioning of energy between the coarse-scale energy fluctuations observed in the CG system and the averaged-out fine scale fluctuations is invariant to molecular weight. Figure $10 \mathrm{~b}$ shows the predicted heat capacities from atomistic and CG simulations with these scaling factors applied via eq 19 for the CG values:

$$
C_{V}=\frac{3}{2} C_{k} N_{C G} k_{\mathrm{B}}+\frac{C_{u}\left\langle\delta U_{C G}{ }^{2}\right\rangle}{k_{\mathrm{B}} T^{2}}+\frac{C_{u k}\left\langle\delta U_{C G} \delta K_{C G}\right\rangle}{k_{\mathrm{B}} T^{2}}
$$

where $N_{C G}$ represents the number of CG beads in the system, $\left\langle\delta U_{C G}{ }^{2}\right\rangle$ is the variance of CG potential energy, and $\left\langle\delta U_{C G} \delta K_{C G}\right\rangle$ is the ensemble averaged arises from the cross correlation of $C G$ potential energy and CG kinetic energy. The sensitivity of the chain length to the heat capacity is somewhat exaggerated in the CG model, however comparison to an experimentally measured value for polyurea of $1.977 \mathrm{~J} \mathrm{~K}^{-1} \mathrm{~cm}^{-3}$ is quite reasonable. ${ }^{22}$

Next, we compare the global structure of the polyurea systems computed using CG and atomistic models, in terms of chain conformations and overall morphology of the system. No attempt is made to compare the local structure from the CG and atomistic models because the IBI parametrization automatically leads to preservation of the local structure of the atomistic system, as noted by the excellent match between the CG and atomistic RDFs (Figure 8). We characterize chain conformations in terms of the distribution of chain end-to-end distances $P\left(d_{\mathrm{ee}}\right)$, where the end-to-end distance $d_{\mathrm{ee}} \equiv\left|\mathbf{r}_{\mathrm{N}}(t)-\mathbf{r}_{1}(t)\right|$. We also qualitatively characterize the overall morphology in terms of the size and shape of microphase-separated domains.

To characterize the effect of the chain length on end-to-end distances in polyurea, five different CG systems of increasing chain length are simulated, with the results shown in Figure 11a. Because of the computational expense in generating equilibrium configurations with all-atom models, only 1-block polyurea end-to-end distribution is computed as verification of the CG model, which is shown in Figure 11b. Because of the slow diffusion in the atomistic system, the end-to-end distributions deviate very slowly from their initial state, and therefore sufficient sampling is challenging even for this short chain
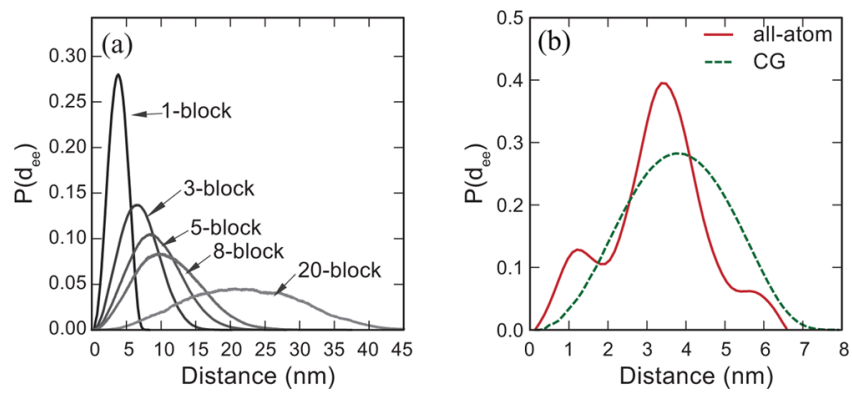

Figure 11. (a) Predicted distributions of chain end-to-end distances $P\left(d_{\mathrm{ee}}\right)$ from CG MD simulations with increasing chain lengths, and (b) Comparison of $P\left(d_{\mathrm{ee}}\right)$ for 1-block systems obtained from all-atom $\mathrm{MD}$ and CG MD simulations.

Table 2. Summary of Expected Value $\left\langle d_{\mathrm{ee}}\right\rangle$ for Different CG and All-Atom MD Systems

no. of blocks
1
3
5
8
20

$\begin{array}{cc}\text { all-atom }(\mathrm{nm}) & \text { CG }(\mathrm{nm}) \\ 3.13 & 3.79 \\ - & 6.99 \\ - & 9.28 \\ - & 11.19 \\ - & 22.17\end{array}$

system. Table 2 summarizes the calculated expected value $\left\langle d_{\mathrm{ee}}\right\rangle$ for different CG systems and the single-block fully atomistic system showing reasonable agreement between the CG and allatom systems for the 1-block polyurea chains. The dependence of the average end-to-end distance with chain length follows a power law with an exponent of 0.577 consistent with the theoretical scaling of 0.588 obtained for three-dimensional random walk chains in good solvent. ${ }^{39}$

To characterize the phase morphology of polyurea, we simulate $\left(\mathrm{H}_{2} \mathrm{~S}_{14}\right)_{1}$ and $\left(\mathrm{H}_{2} \mathrm{~S}_{14}\right)_{20} \mathrm{CG}$ polyurea systems containing 128000 beads in a cubic simulation box that has an edge length of $26.5 \mathrm{~nm}$. As a comparison, we also simulate a fully atomistic system containing 160 1-block chains, equivalent to 2,560 CG beads, in a $7.2 \mathrm{~nm}$ cubic simulation box. Figure 12a shows the initially random configuration of the single block CG system. After $200 \mathrm{~ns}$ of simulation time, the resulting microstructures for the $\left(\mathrm{H}_{2} \mathrm{~S}_{14}\right)_{1}$ and $\left(\mathrm{H}_{2} \mathrm{~S}_{14}\right)_{20}$ systems can be seen in Figure 12, parts $b$ and $c$. The initial and final states of the atomistic system are shown in Figure 12, parts $\mathrm{d}$ and e. The atomistic system is equilibrated for only $32 \mathrm{~ns}$, but at elevated temperatures as previously described. To more quantitatively compare the morphology of the CG and all-atom systems, and to investigate the effect of the number of blocks, we computed the number and sizes of segregated hard domains for single block and 20 block CG systems. Clusters are identified by grouping all neighboring hard beads with a neighbor threshold distance of $7.5 \AA$ A. A clear difference between the morphology of the $\left(\mathrm{H}_{2} \mathrm{~S}_{14}\right)_{1}$ and $\left(\mathrm{H}_{2} \mathrm{~S}_{14}\right)_{20}$ systems can be observed as the single block polyurea chains tend to aggregate into a fewer number of larger clusters as observed by Arman et al. ${ }^{9}$ These results are plotted in Figure 13 for the CG systems. Since the fully atomistic simulation box contains only $2 \%$ of the volume of the CG simulation box, quantitative analysis of the number and size of the segregated hard domains is not meaningful, although qualitatively, the clustering of hard domain segments is similar to the CG models. Moreover, fully atomistic simulations of phase segregation in polyurea for quantitative predictions would require prohibitive computational effort. 


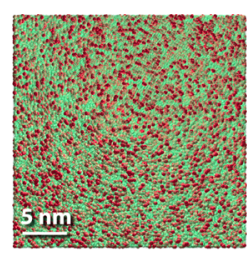

(a) $\mathrm{CG}$, 1-block, $0 \mathrm{~ns}$

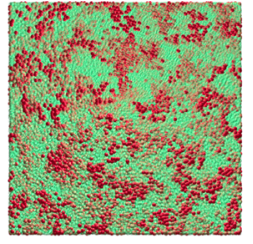

(b) CG, 1-block, $200 \mathrm{~ns}$

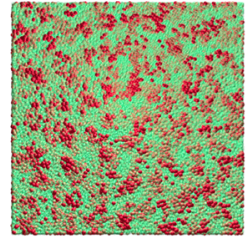

(c) CG, 20-block, $200 \mathrm{~ns}$

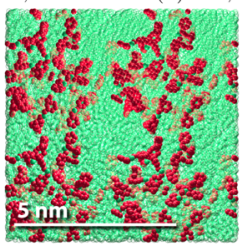

(d) all-atom, 1-block, 0 ns

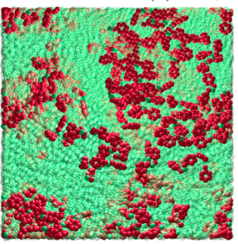

(e) all-atom, 1-block, $32 \mathrm{~ns}$
Figure 12. Morphologies of CG and all-atom polyurea systems before and at the end of the simulation runs: (a) initial and (b) final morphology of 1-block CG chains; (c) final morphology of 20-block CG chains; and (d) initial and (e) final morphology of 1-block allatom chains. Red points represent the hard segments while green points represent the soft segments.

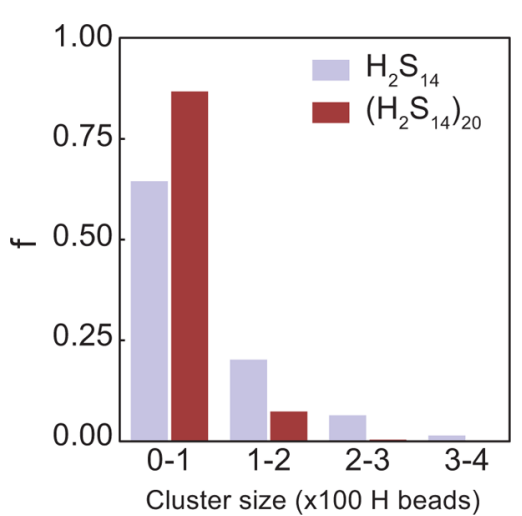

Figure 13. Size distribution of hard domains in terms of the fraction of $\mathrm{H}$ beads within each hard domain for $\mathrm{H}_{2} \mathrm{~S}_{14}$ and $\left(\mathrm{H}_{2} \mathrm{~S}_{14}\right)_{20}$.

Finally, we compare the stress relaxation function $G(t)$ from a one block system computed from both the CG and atomistic models. Since the stress relaxation function computed directly from the autocorrelation of the virial stress via eq 17 is highly noisy, we take running averages of the raw $G(t)$ between $0.9 t$ and $1.1 t$, consistent with previous studies. ${ }^{40}$ Furthermore, the time axis of the CG stress relaxation function must be rescaled to account for the faster dynamics of the CG system due to its smoothed energy landscape and reduced frictional forces acting between molecules. While several authors have suggested the use of a constant scaling factor related to the ratio of selfdiffusivities, ${ }^{41,42}$ we find that this is insufficient to match the profile of the stress relaxation function between the CG and allatom models. Instead we observe that the ratio in the mean square displacement between the two systems is not constant, starting at a value near unity and increasing until reaching an asymptotic value after 100 ns. This trend suggests that at extremely short time spans, the diffusion of the system is limited only by the available thermal energy and momenta of the atoms, and not until longer time scales is motion limited by frictional forces. Figure 14a shows the ratio of mean square displacements from the CG and all-atom MD systems fitted to a time-dependent dynamic rescaling function $f_{t}(t)$, defined as.

$$
f_{t}(t)=1+a\left(1-\mathrm{e}^{-t / \tau}\right)
$$
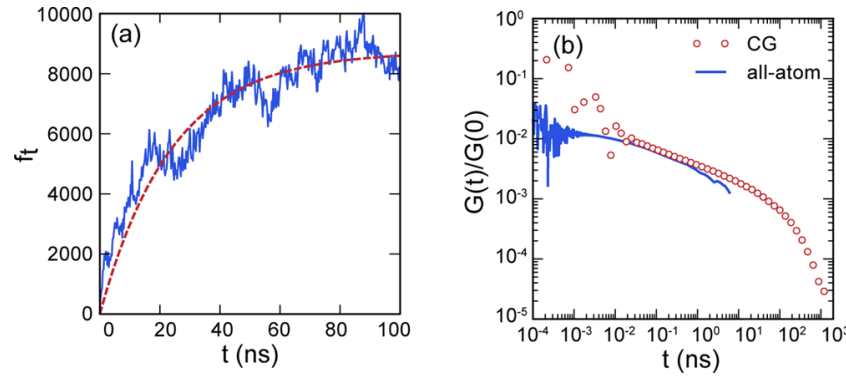

Figure 14. (a) Dynamic scaling factor $f_{\mathrm{t}}$ for $\left(\mathrm{H}_{2} \mathrm{~S}_{14}\right)_{1}$ system. The dashed line shows the exponential fit of $f_{\mathrm{t}}$. (b) Comparison of all-atom $\mathrm{MD}$ and scaled CG stress relaxation spectra $G(t) / G(0)$ for $\left(H_{2} S_{14}\right)_{1}$.

where the constants $a=8,731$ and $\tau=23.8$ ns reflect the long time scale scaling constant and transition time, respectively. Thus, the relaxation spectrum for the CG model is rescaled as

$$
G(t)=G_{C G}\left(t_{C G} \times f_{t}\left(t_{C G}\right)\right)
$$

Figure $14 \mathrm{~b}$ compares the rescaled CG relaxation spectrum for $\left(H_{2} S_{14}\right)_{1}$ with all-atom $\mathrm{MD}$ simulation. At very short time scales, $(t \leq 10 \mathrm{ps})$, the stress relaxation function of the all-atom system decays much faster than that of the CG system because some of the atomic-scale relaxation mechanisms, such as damped oscillations of collective bond and bending angle vibrations, are not represented in the CG model. At intermediate time scales ( $10 \mathrm{ps} \leq t \leq 1 \mathrm{~ns}$ ) the agreement between the two models is excellent. Beyond $1 \mathrm{~ns}$ sufficient sampling of the all-atom MD model becomes increasing computationally expensive and the autocorrelation of the stress decays rapidly with insufficient sampling. However, the efficiency of the CG model allows for sufficient sampling well past $1000 \mathrm{~ns}$, far beyond the practical range of all-atom MD simulations.

\section{DISCUSSION}

We have developed a CG model of polyurea, composed of a series of "soft" and "hard" CG beads that represent the flexible aliphatic and more rigid aromatic moieties connected by urea linkages, respectively. The interaction potential for the CG beads is calibrated with the iterative Boltzmann inversion method to match structural distributions obtained from atomistic MD simulations of oligomeric polyurea systems. Additional structural and physical properties, such as end-to-end distributions, density, and heat capacity are compared between the CG and atomistic models to verify that the CG model is representative of the atomistic $\mathrm{MD}$ model. The CG model allows prediction of stress relaxation in oligomeric systems at microsecond time scales due to the increased efficiency and faster dynamics of the CG system. By rescaling time in the CG simulation by the ratio of mean square displacements in allatom and CG MD simulations, quantitative agreement with the relaxation spectrum predicted from atomic $\mathrm{MD}$ simulations is achieved. Provided that this time rescaling function is insensitive to the length of the polymer chain, this approach may allow for quantitative predictions of stress relaxation in polymers with realistic molecular weights.

For atomistic $\mathrm{MD}$ simulations of polyurea, it is evident that even short oligomeric chains require a significant amount of computational time to reach a well-equilibrated configuration. For instance, systems composed of a single hard segment connected to a chain of 14 tetramethylene oxide monomers require at least $32 \mathrm{~ns}$ of equilibration time at elevated temperatures to 
reach a steady state distribution. The most significant change observed during the equilibration of such oligomeric polyurea systems is the increase in the height of the peak in the $\mathrm{H} / \mathrm{H}$ pair radial distribution function. Since this height corresponds to the depth of the energy well describing mutual attraction between hard beads, calibration of a poorly equilibrated system with the IBI method will result in under-prediction of hard segment attraction and produce a system less likely to phasesegregate and ultimately not representative of polyurea. CG simulations conducted to compare the phase segregation of systems containing short oligomeric polyurea chains with systems containing longer chains produced similar results compared to the bead-spring model developed by Arman et al., ${ }^{9}$ where short oligomers produced larger clustered hard domains than did longer multiblock chains.

However, even with CG models, the simulation box sizes employed in this study are still far too small to reproduce the experimentally observed morphological features of polyurea, which can be as large as $100 \mathrm{~nm}$ in size. ${ }^{6}$ To address this challenge, a higher degree of coarse-graining would be required, though CG models that amalgamate greater numbers of atoms within a single bead will have less accurate dynamics due their smoother energy landscapes, which results in reduced intermolecular friction. Furthermore, as the number of CG degrees of freedom decrease, the distance between bonded CG beads increases, allowing for unphysical crossings of polymer chains, making simulations inaccurate for prediction of dynamical and mechanical properties. Therefore, optimal CG models are likely to be specialized for each application. For predictions of morphology at realistic length scales, inaccurate dynamics of highly coarse-grained models are irrelevant, or perhaps even an advantage as the system can find the equilibrium state more rapidly. Conversely, for computing rheological properties, CG models would require higher detail in their mappings.

With copolymers that exhibit significant phase segregation, optimization of all pair potentials simultaneously with iterative Boltzmann inversion appears to be more robust than individually optimizing each pair potential sequentially, due to the interdependencies between pair interactions. For example, in our polyurea CG model, the $\mathrm{H} / \mathrm{H}$ and $\mathrm{H} / \mathrm{S}$ interactions are correlated, as strong aggregation of the $\mathrm{H}$ beads automatically leads to some aggregation between the $\mathrm{S}$ and $\mathrm{H}$ beads, due to chain connectivity. Therefore, modifying the $\mathrm{H} / \mathrm{H}$ potential alters both the $\mathrm{H} / \mathrm{H}$ and $\mathrm{H} / \mathrm{S}$ radial distribution functions. To improve the stability of the IBI method, corrections to the pair potentials are scaled by an additional parameter, $\alpha$, to reduce oscillations, mitigate strongly coupled interactions, and improve convergence to the target pair distributions. The optimal scaling parameter depends strongly on the underlying atomistic chemistry and CG mapping. In this work, we selected an initial value of unity and iteratively halved its value until the number of iterations required for convergence was minimized; we used the same scaling parameter for all pair combinations. A scaling factor of 0.05 is nearly optimal for our CG mapping of polyurea and excellent convergence of the target radial distribution functions is achieved within 100 iterations. While efforts to determine effective CG potentials for a phase segregating polymer blends of polyisoprene (PI) and polystyrene (PS) have been previously reported, ${ }^{43,44}$ the degree of independence between the PS/PS, PS/PI, and PI/PI pair potentials was far less than that observed here for polyurea. For instance, the peak in the radial distribution function of the polyurea $\mathrm{CG} \mathrm{H} / \mathrm{H}$ beads is four times higher than the reported peak in the PI/PI or PS/PS radial distribution functions.

Lastly, the computational efficiency gained from CG models is substantial, allowing $\mathrm{MD}$ simulations to probe the dynamics of polymers at time scales relevant to shock propagation. Fully atomistic NVT simulations of polyurea containing 10k atoms, executed in parallel on 64 CPU cores (Intel Xeon E5-2670 processors) progressed at a rate of 21.9 ns per day, while equivalent CG simulations progressed at $17.9 \mu$ s per day on 32 cores, i.e., a speedup of 3 orders of magnitude. With the additional speed up in dynamics of the CG system, the CG model can probe relaxation phenomena in polyurea with an overall effective speedup of 6-7 orders of magnitude compared to the atomistic MD models.

\section{AUTHOR INFORMATION}

\section{Corresponding Author}

*(J.O.) E-mail: j-oswald@asu.edu.

\section{Notes}

The authors declare no competing financial interest.

\section{ACKNOWLEDGMENTS}

The authors gratefully acknowledge support from the Office of Naval Research under Grant Number N00014-09-1-1126 to University of California, San Diego. We also thank Greg Hattemer for help with viscoelasticity calculations and Dr. Roshdy Barsoum, Prof. Sia Nemat-Nasser, Prof. Zhibin Guan, and Dr. Alireza Amirkhizi for many fruitful discussions.

\section{REFERENCES}

(1) Das, S.; Cox, D. F.; Wilkes, G. L.; Klinedinst, D. B.; Yilgor, I.; Yilgor, E.; Beyer, F. L. J. Macromol. Scie., Part B: Phys. 2007, 46, 853875.

(2) Chattopadhyay, D.; Raju, K. Prog. Polym. Sci. 2007, 32, 352-418.

(3) Barsoum, R. G. S. Challenges in Mechanics of Time-Dependent Materials and Processes in Conventional and Multifunctional Materials. In Proceedings of the 2012 Annual Conference on Experimental and Applied Mechanics; Antoun, B., Qi, H. J., Hall, R., Tandon, G. P., Lu, H., Lu, C., Eds.; Springer: New York, 2013; Vol. 2.

(4) Tekalur, S.; Shukla, A.; Shivakumar, K. Compos. Struct. 2008, 84, 271-281.

(5) Gardner, N.; Wang, E.; Kumar, P.; Shukla, A. Exp. Mech. 2012, 52, 119-133.

(6) Castagna, A.; Pangon, A.; Choi, T.; Dillon, G.; Runt, J. Macromolecules 2012, 45, 8438-8444.

(7) Lieser, G.; Fischer, E. W.; Ibel, K. J. Polym. Sci., Part C: Polym. Lett. 1975, 13, 39-43.

(8) Pearson, D. S.; Strate, G. V.; von Meerwall; Schilling, F. C. Macromolecules 1987, 20, 1133-1141.

(9) Arman, B.; Srinivas Reddy, A.; Arya, G. Macromolecules 2012, 45, 3247.

(10) Grujicic, M.; Pandurangan, B.; King, A. E.; Runt, J.; Tarter, J.; Dillon, G. J. Mater. Sci. 2010, 46, 1767-1779.

(11) Chantawansri, T. L.; Sliozberg, Y. R; Andzelm, J. W.; Hsieh, A. J. Polymer 2012, 53, 4512-4524.

(12) Kremer, K.; Grest, G. S. J. Chem. Phys. 1990, 92, 5057.

(13) Li, Y.; Abberton, B. C.; Kröger, M.; Liu, W. K. Polymers 2013, 5, $751-832$.

(14) Reith, D.; Meyer, H.; Müller-Plathe, F. Macromolecules 2001, 34, 2335-2345.

(15) Reith, D.; Pütz, M.; Müller-Plathe, F. J. Comput. Chem. 2003, 24, 1624-1636.

(16) Müller-Plathe, F. ChemPhysChem 2002, 3, 754-769.

(17) Milano, G.; Müller-Plathe, F. J. Phys. Chem. B 2005, 109, 18609-18619. 
(18) Bayramoglu, B.; Faller, R. Bull. Am. Phys. Soc. 2011, 56, BAPS.2011.MAR.C1.80.

(19) Maurel, G.; Schnell, B.; Goujon, F.; Couty, M.; Malfreyt, P. J.

Chem. Theory Comput. 2012, 8, 4570-4579.

(20) Kamio, K.; Moorthi, K.; Theodorou, D. N. Macromolecules 2007, 40, 710-722.

(21) Yi, J.; Boyce, M.; Lee, G.; Balizer, E. Polymer 2006, 47, 319-329.

(22) Amirkhizi, A.; Isaacs, J.; McGee, J.; Nemat-Nasser, S. Philos. Mag. 2006, 86, 5847-5866.

(23) Fragiadakis, D.; Gamache, R.; Bogoslovov, R; Roland, C. Polymer 2010, 51, 178-184.

(24) Roland, C.; Casalini, R. Polymer 2007, 48, 5747-5752.

(25) Domeier, L. Urethane Elastomers: Development of TDI-Free Replacement Materials for EN-7; 2000; http://www.osti.gov/scitech/ servlets/purl/766630.

(26) Accelrys, Accelrys 2012. http://accelrys.com/products/ materials-studio/.

(27) Plimpton, S.; Crozier, P.; Thompson, A. LAMMPS; Sandia National Laboratories: Sandia, NM, 2007.

(28) Sun, H. J. Phys. Chem. B 1998, 102, 7338-7364.

(29) Hwang, M.; Stockfisch, T.; Hagler, A. J. Am. Chem. Soc. 1994, $116,2515-2525$.

(30) McQuaid, M.; Sun, H.; Rigby, D. J. Comput. Chem. 2003, 25, $61-71$.

(31) Frenkel, D.; Smit, B. Understanding Molecular Simulation: From Algorithms to Applications; Computational Science; Elsevier Science: Amsterdam, 2001.

(32) Faller, R. Polymer 2004, 45, 3869-3876.

(33) Ruhle, V.; Junghans, C.; Lukyanov, A.; Kremer, K.; Andrienko, D. J. Chem. Theory Comput. 2009, 5, 3211-3223.

(34) Eslami, H.; Karimi-Varzaneh, H. A.; Müller-Plathe, F. Macromolecules 2011, 44, 3117-3128.

(35) Plimpton, S. J. Comput. Phys. 1995, 117, 1-17.

(36) Allen, M.; Tildesley, D. Computer simulation of liquids.; Oxford Science Publications; Oxford University Press: Oxford, U.K., 1989.

(37) Zhou, Y.; Karplus, M.; Wichert, J. M.; Hall, C. K. J. Chem. Phys. 1997, 107, 10691.

(38) Amini, M.; Simon, J.; Nemat-Nasser, S. Mech. Mater. 2010, 42, 615-627.

(39) Rubinstein, M.; Colby, R. H. Polymer Physics; OUP: Oxford, U.K., 2003.

(40) Sen, S.; Kumar, S. K.; Keblinski, P. Macromolecules 2005, 38, 650-653.

(41) Fritz, D.; Koschke, K.; Harmandaris, V. A.; van der Vegt, N. F.; Kremer, K. Phys. Chem. Chem. Phys. 2011, 13, 10412-10420.

(42) Fritz, D.; Herbers, C. R.; Kremer, K.; van der Vegt, N. F. Soft Matter 2009, 5, 4556-4563.

(43) Sun, Q.; Faller, R. J. Chem. Theory Comput. 2006, 2, 607-615.

(44) Sun, Q.; Faller, R. J. Chem. Phys. 2007, 126, 144908. 